Article

\title{
Assimilation of Typhoon Wind Field Retrieved from Scatterometer and SAR Based on the Huber Norm Quality Control
}

\author{
Boheng Duan ${ }^{1, *}(\mathbb{D})$, Weimin Zhang ${ }^{1}$, Xiaofeng Yang ${ }^{2,3}{ }^{(D)}$, Haijin Dai ${ }^{1}$ and $\mathrm{Yi} \mathrm{Yu}^{1}$ \\ 1 Academy of Ocean Science and Engineering, National University of Defense Technology, Changsha 410073, \\ China; wmzhang104@139.com (W.Z.); hj_dai@nudt.edu.cn (H.D.); yuyi2019@nudt.edu.cn (Y.Y.) \\ 2 State Key Laboratory of Remote Sensing Science, Chinese Academy of Sciences, Beijing 100101, China; \\ yangxf@radi.ac.cn \\ 3 The Key Laboratory for Earth Observation of Hainan Province, Sanya 572029, China \\ * Correspondence: bhduan@foxmail.com; Tel.: +86-156-7482-7043
}

Received: 17 June 2017 ; Accepted: 20 September 2017 ; Published: 23 September 2017

\begin{abstract}
Observations of sea surface wind field are critical for typhoon prediction. The scatterometer observation is one of the most important sources of sea surface winds, which provides both wind speed and wind direction information. However, the spatial resolution of scatterometer wind is low. Synthetic Aperture Radar (SAR) can provide a more detailed wind structure of the tropical cyclone. In addition, the cross-polarization observation of SAR can provide more detailed information of high speed wind $\left(>25 \mathrm{~m} \cdot \mathrm{s}^{-1}\right)$ than the scatterometer. Nevertheless, due to the narrow swath of SAR, the number of retrieved sea surface wind data used in the data assimilation is limited, and another limitation of SAR wind observation is that it does not provide true wind direction information. In this paper, the joint assimilation of the Advanced Scatterometer (ASCAT) wind and Sentinel-1 SAR wind was investigated. Another limitation in the current operational typhoon prediction is the inefficient quality control (QC) method used in the data assimilation since a large number of high speed wind observations was rejected by the traditional Gaussian distribution QC. We introduce the Huber norm distribution quality control (QC) into the data assimilation successfully. A numerical simulation experiment of typhoon by Lionrock (2016) is conducted to test the proposed method. The experimental results showed that the new quality control scheme not only greatly increases the availability of wind data in the area of the typhoon center, but also improves the typhoon track prediction, as well as the intensity prediction. The joint assimilation of scatterometer and SAR winds does have a positive impact on the typhoon prediction.
\end{abstract}

Keywords: tropical cyclone; scatterometer wind; SAR wind; data assimilation; quality control; Huber norm

\section{Introduction}

The demand for more accurate predictions of tropical typhoons is increasing in order to minimize losses and destruction. One primary objective is to enhance the observation targeting and observability of cyclones. Satellite observations can effectively compensate for the shortcomings of traditional methods of sea surface measurement and provide all-weather observation over the sea surface, which is of great significance to improve the numerical prediction of strong convective weather in the marine area. The spaceborne scatterometer observes the backscattering caused by the sea surface roughness, and then, the sea surface wind can be retrieved. ASCAT is one of the instruments carried on-board the Meteorological Operational (Metop) polar satellites launched by the European Space Agency (ESA) and operated by the European organization for the exploitation of Meteorological 
Satellites (EUMETSAT) [1]. Its operating frequency is C-band $(5.255 \mathrm{GHz})$, so the effects of clouds and precipitation in the observation are small. ASCAT has two swaths, and each has a scanning width of $550 \mathrm{~km}$. It can achieve a daily quasi-global coverage.

Scatterometer data were first used in a numerical weather forecasting operational system in 1996, when the European Center for Medium-Range Weather Forecasts (ECMWF) incorporated ERS-1 (European remote sensing satellite) scatterometer data into its global three-dimensional variational system [2]. Previous works have shown that scatterometer data have significant impacts on weather forecasting and climate monitoring [3-10]. Especially, it has been demonstrated useful in the prediction of tropical cyclones [5] and extratropical cyclones [4]. ASCAT surface wind data have been used in many forecasting operational organizations such as the ECMWF, the United Kingdom's National Weather Service (Met Office), the National Weather Service of France (Meteo-France) and Environment Canada. In July 2009, the Japan Meteorological Agency (JMA) began to use ASCAT data for the global spectrum model (GSM) and found that the ASCAT wind can capture the development of the low pressure system and improve the prediction precision. Hersbach (2010) pointed out that the neutral wind retrieved by ASCAT had a positive effect on the ECMWF forecasting system [11]. In 2011, Li evaluated the role of the ASCAT wind in the global data assimilation system of the NCEP (National Centers for Environmental Prediction), and the results showed that ocean surface wind of ASCAT has a positive effect on the forecast of wind and temperature [1].

Spaceborne SAR systems are an important data source for sea surface monitoring. In 1978, the United States of America launched the first synthetic aperture radar satellite SEASAT. Since then, many countries have begun to carry out the study of the spaceborne SAR system vigorously. Most of the systems used the single-band, single polarization imaging radar systems in the early stage, such as ESA's ERS-1/2, Canada's Radarsat-1, and so on. At the beginning of the 20th Century, the spaceborne SAR system was developed into multi-band, multi-polarization and multi-mode. The Envisat satellite launched by ESA in 2002, with the multi-polarization interferometric imaging model, is widely used in natural disaster monitoring and resource and environmental survey. The launch of the Japanese ALOS (Advanced Land Observing Satellite) in 2006 aimed to provide full polarimetric SAR data. The launch of the COSMO-SkyMed (Constellationof small Satellites for the Mediterranean basin Observation) satellite in 2006 by Italy made the satellite resolution increase to $3 \mathrm{~m}$ or even $1 \mathrm{~m}$. In 2007, Germany launched the TerraSAR-X satellite with a revisit cycle of 11 days, which greatly improved the coherence of interference data. Canada's Radarsat-2 satellite has been able to provide full polarimetric image with high-resolution since 2007. The Sentinel-1 was launched by ESA in April 2014 to provide data services for more users with its wide range of multi-mode, multi-application features. The Sentinel- 1 carries a single C-band synthetic aperture radar instrument operating at a center frequency of $5.405 \mathrm{GHz}$. Its extra-wide (EW) swath mode data can cover a wide area of $400 \mathrm{~km}$ at a medium resolution of $20 \mathrm{~m}$ by $40 \mathrm{~m}$ on the ground. It also has the capability of dual polarization, a short revisit cycle and rapid productization. Using pre-programmed, conflict-free operation mode, the Sentinel-1 can track and monitor a typhoon center dynamically.

A variety of meteorological hydrological elements can be retrieved by SAR observation, and it has been widely used in data assimilation in recent years. The first attempt to sequentially assimilate ESA's ERS SAR estimations of surface soil moisture was conducted in 2003 [12]. Matgen, P. (2010) presented a new concept for the sequential assimilation of SAR-derived water stages into coupled hydrologic-hydraulic models [13]. Scott, K. A. (2015) investigated the assimilation of binary observations calculated from SAR images of sea ice [14]. Phan, X. V. (2014) introduced a variational data assimilation scheme coupling TerraSAR-X radiometric data with the snowpack evolution model Crocus, and the results indicated that X-band SAR data can be taken into account to modify the evolution of snowpack simulated by Crocus [15]. Pichelli, E. (2015) developed a technique to retrieve integrated water vapor from interferometric synthetic aperture radar (InSAR) data, and the computation of statistical indices shows that the InSAR assimilation improves the forecast of weak to moderate precipitation [16]. Advanced Synthetic Aperture Radar (ASAR) wide swath data were used to measure 
soil moisture by [17], and they have sufficient resolution to allow soil moisture variations due to local topography to be detected, which helps to take into account the spatial heterogeneity of hydrological processes. Another important use of SAR is the monitoring of typhoons and the study of typhoon structure [18-24]. Many studies have also been carried out about the retrieval of ocean winds from SAR image [25-28], the and estimation of the retrieved wind shows that the SAR wind has an ideal accuracy [29-32]. However, the use of SAR-retrieved wind in numerical weather forecasts is a relatively new area, especially for typhoon prediction.

Quality control of observation is an indispensable process for data assimilation [33]. It ensures that the wrong observations are removed before assimilation, which would otherwise result in inaccurate analysis [34]. In general, the deviation of background (b) and observation (o) is used as a basis for evaluating the quality of the data. It is generally believed that the observation error distribution satisfies the Gaussian distribution, assuming that the background error is a Gaussian distribution, and then, the distribution of deviation should also be satisfied with the Gaussian distribution. However, according to the statistical results, the distribution of deviation for many observations does not strictly follow the Gaussian distribution. The traditional Gaussian distribution QC method carries out strict threshold control by the magnitude of the deviation. However, large deviation between the observation and background does not mean that the observation is wrong. In extreme weather conditions, observation and background tend to have a larger deviation, and the use of traditional Gaussian distribution QC will result in rejection of a large number of effective observations. In fact, the deviation often complies with a Huber norm distribution [35,36]. In 2009, the Huber norm-based QC method was applied to both the deterministic and ensemble forecasting system at ECMWF, but only for conventional observations. Unlike traditional QC methods, this method takes appropriate weights based on the magnitude of deviation from the observation and background, making it possible to utilize more observations. Based on the Huber norm QC scheme, this paper calculates the transition point of the Huber norm distribution and adjusts the weight of the observation error for the wind data.

In this paper, a data assimilation scheme is proposed to jointly use SAR and scatterometer retrieved winds in the Weather Research and Forecasting (WRF) model. The improved Huber norm QC method is also introduced. Following this Introduction, a brief description of the ASCAT scatterometer wind and the Sentinel-1 SAR wind is given in Section 2. Section 3 introduces the detailed scheme of the Huber norm QC. In Section 4, we use a numerical simulation experiment of the typhoon Lionrock case to test the proposed method. Finally, the conclusion is given in Section 5.

\section{Retrieved Wind of Scatterometer and SAR}

\subsection{ASCAT Wind}

Two sets of three antennas are used in the ASCAT to generate radar beams looking 45 degrees forward, sideways and 45 degrees backwards with respect to the satellite's flight direction, on both sides of the satellite ground track. For each wind vector cell (WVC), ASCAT obtains three independent backscatter measurements using the three different viewing directions, separated by a short time delay. Then, the surface wind speed and direction can be obtained by using these 'triplets' within a geophysical model functions (GMF). The wind product we used in the paper is obtained through the processing of scatterometer data originating from the ASCAT instrument of EUMETSAT's Metop-B satellite with a resolution of $12.5 \mathrm{~km}$. Figure 1 shows the ASCAT wind field of the center of the typhoon "Lionrock" at 9 a.m. on 29 August 2016. However, the wind field does not cover a complete typhoon eye due to the limitation of the ASCAT swath. 


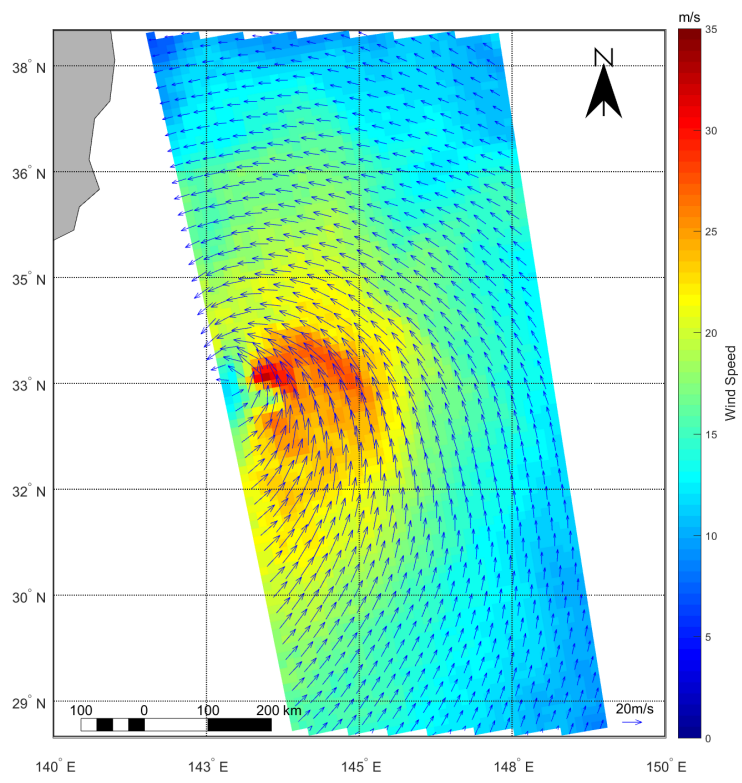

Figure 1. ASCAT wind field of typhoon "LionRock" with a resolution of $12.5 \mathrm{~km}$.

\subsection{SAR Wind of Satellite Sentinel-1}

There exists an undisclosed problem for the retrieval of wind through SAR observation, that is one SAR observation corresponds to numerous wind speed and wind direction solutions through the geophysical model function (GMF). To solve the problem of SAR wind inversion, new information, which can be obtained from the SAR image itself or from the numerical forecast model or buoy observation data, must be added. In this paper, the wind direction information used for the wind inversion of Sentinel-1 SAR data comes from interpolation of the numerical forecast model (NCEP/GFS), and then, the wind speed can be retrieved by using the C-2PO (C-band Cross-Polarization Ocean) GMF [37].

In this study, a Sentinel-1A EWswath mode dual-polarization (VV/VH) SAR image is used to retrieve sea surface wind speed (see Figure 2). Its overpass time was 20:36:44 UTC on 29 August 2016. The observation using VV polarization can get a good signal to noise ratio (SNR) in the low speed wind conditions, and the accuracy of the retrieved wind is high. However, either the scatterometer nor the SAR observation using VV polarization are sensitive to the physical feedback of the sea surface under high speed wind conditions (wind speed greater than $25 \mathrm{~m} \cdot \mathrm{s}^{-1}$ ), and the detection signal would reach a level of saturation. In other words, the effective range of the wind speed inversion for scatterometer and SAR observation with VV polarization is limited to $25 \mathrm{~m} \cdot \mathrm{s}^{-1}$. The SAR observation using $\mathrm{VH}$ polarization is sensitive to high speed wind conditions, and it can retrieve the wind speed greater than $25 \mathrm{~m} \cdot \mathrm{s}^{-1}$, but the SNR is low under low speed wind conditions. As shown in Figure $2 \mathrm{~b}$, $\mathrm{VH}$ polarization observation of the typhoon region can get more high speed wind information, but there is a large amount of noise in the non-high speed wind region.

In order to combine the advantages of two kinds of polarization modes, we use a simple linear weighted method to composite these two retrieved wind fields. The main idea is that more weight is given to the $\mathrm{VV}$ polarization retrieved wind when the wind speed (we use the $v_{V V}$ as the reference wind speed) is less than $25 \mathrm{~m} \cdot \mathrm{s}^{-1}$, on the other hand, more weight for the $\mathrm{VH}$ polarization retrieved wind when the wind speed is greater than $25 \mathrm{~m} \cdot \mathrm{s}^{-1}$. The specific expression is as follows:

$$
v_{S}= \begin{cases}\left(1-\lambda_{1}\right) \cdot v_{V V}+\lambda_{1} \cdot v_{V H} & \text { if } v_{V V} \leq 25 \mathrm{~m} \cdot \mathrm{s}^{-1} \\ \left(1-\lambda_{2}\right) \cdot v_{V V}+\lambda_{2} \cdot v_{V H} & \text { if } v_{V V}>25 \mathrm{~m} \cdot \mathrm{s}^{-1}, \text { and } 25 \mathrm{~m} \cdot \mathrm{s}^{-1}<v_{V H}<35 \mathrm{~m} \cdot \mathrm{s}^{-1} \\ v_{V H} & \text { if } v_{V V}>25 \mathrm{~m} \cdot \mathrm{s}^{-1}, \text { and } v_{V H} \geq 35 \mathrm{~m} \cdot \mathrm{s}^{-1}\end{cases}
$$


where $v_{V V}, v_{V H}$ and $v_{S}$ represent $\mathrm{VV}$ polarization wind speed, $\mathrm{VH}$ polarization wind speed and synthetic wind speed, respectively; $\lambda_{1}$ and $\lambda_{2}$ are the linear functions of the wind speed, where $\lambda_{1} \in[0,0.5], \lambda_{2} \in[0.5,1]$. The setup of $\lambda_{1}$ and $\lambda_{2}$ makes sure that the VV polarization retrieved wind gets more weight when $v_{V V} \leq 25 \mathrm{~m} \cdot \mathrm{s}^{-1}$ and the VH polarization retrieved wind gets more weight when $v_{V V}>25 \mathrm{~m} \cdot \mathrm{s}^{-1}$. As shown in Figure 2c, the synthesized wind field not only preserves the low speed wind information of VV polarization observation, but also introduces the high speed wind information of $\mathrm{VH}$ polarization observation.

Compared to the ASCAT wind field, the SAR wind field has a higher resolution, so that a fine typhoon structure can be demonstrated. It can also be seen from the figure that there are still some discontinuities among different radar beams in the SAR wind field. However, we can see later that these discontinuities can be removed by thinning the observations.

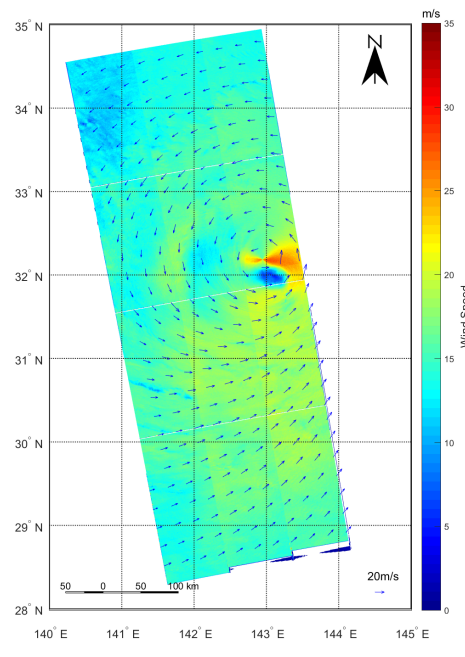

(a)

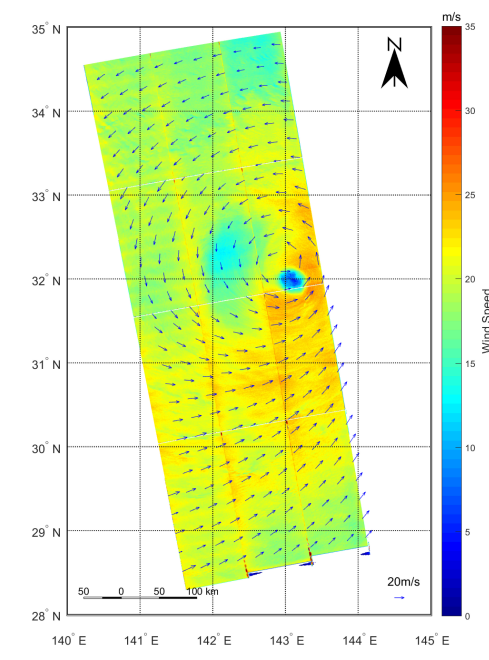

(b)

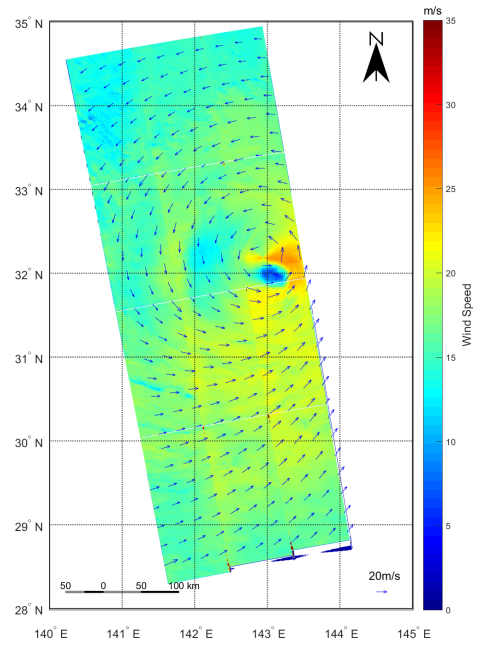

(c)

Figure 2. SAR wind field of satellite Sentinel-1 in the typhoon "Lionrock" region with a resolution of $500 \mathrm{~m}$ (the arrows here are thinned for clarity): (a) retrieved wind using VV polarization observation; (b) retrieved wind using $\mathrm{VH}$ polarization observation; (c) synthetic wind combined with $\mathrm{VV}$ and $\mathrm{VH}$ polarization retrieved wind.

\subsection{Joint Wind Field}

In order to obtain a complete typhoon wind field, we combine the ASCAT wind field with the Sentinel-1 SAR synthetic wind field. First, we thin the two wind fields to $25 \mathrm{~km}$ by sampling to accommodate the needs of the assimilation system. For the ASCAT wind field with a resolution of $12.5 \mathrm{~km}$, we just need to sample every two winds from both zonal and meridional directions. Additionally, we sample every 50 winds for SAR synthetic wind field with a resolution of $500 \mathrm{~m}$. Then, the overlapped winds of the SAR synthesis wind field with the ASCAT wind field are removed. Since the swath of the SAR synthetic wind field is smaller than that of the ASCAT and has no real wind direction information, it is mainly used as a complement to the ASCAT wind field. The joint wind field is illustrated in Figure 3, which forms a complete wind observation of typhoon "Lionrock". It can also be seen from the figure that the discontinuities among different radar beams in the SAR wind field are greatly eliminated by the thinning process. 


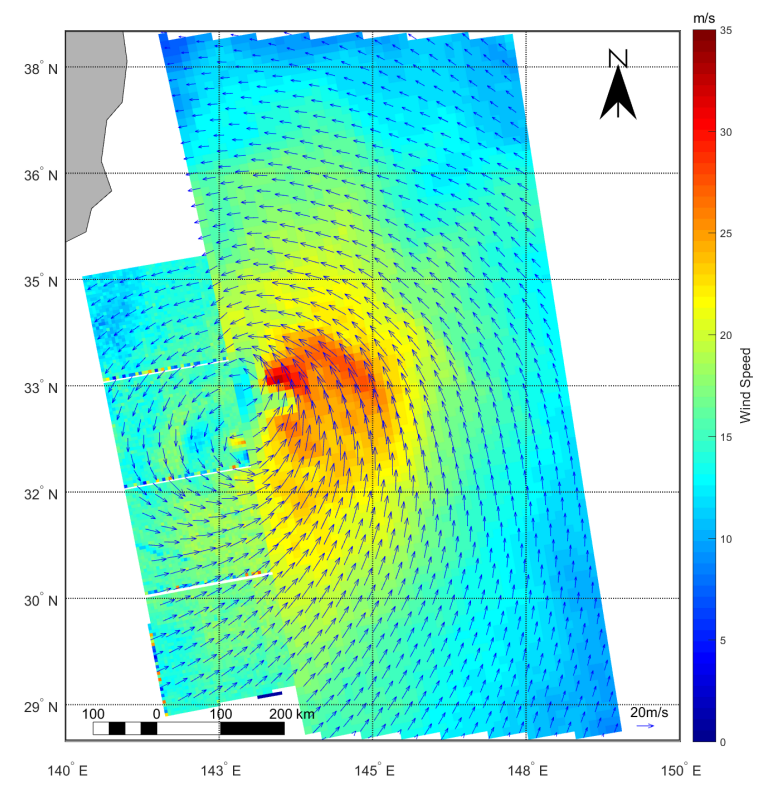

Figure 3. Joint wind field of typhoon "Lionrock" with a resolution of $25 \mathrm{~km}$.

\section{Huber Norm Distribution QC}

\subsection{Gaussian Distribution QC Scheme}

The traditional Gaussian distribution QC assumes that the deviation between observation and background satisfies the Gaussian distribution. Since observation with a big deviation will cause the instability of the assimilation process, the observation that goes into the assimilation process should be strictly controlled. In the Gaussian distribution QC, observation that satisfies $(o-b)^{2}<\alpha^{2}\left(\sigma_{o}^{2}+\sigma_{b}^{2}\right)$ can be received by the assimilation system, where $\sigma_{o}$ and $\sigma_{b}$ represent the standard deviation of the error of observation and background error, respectively (it should be noted that observation errors inside the assimilation system are different from the errors of observation here). In the assimilation system we used in this study, $\alpha$ is set to five, which means observation with deviation more than five-times the observation error was rejected. However, in extreme weather conditions, although observation and background tend to have a large deviation, it does not mean that the observation has a gross error, since the background sometimes also has large errors. Most of the high speed wind observation using the traditional Gaussian distribution QC were rejected before the assimilation, as shown in Figure 4, which shows the joint typhoon wind field after the traditional Gaussian distribution QC. In this paper, the magnitude of the observation error is defined as $2 \mathrm{~m} \cdot \mathrm{s}^{-1}$ (namely, $\sqrt{\left(\sigma_{o}^{2}+\sigma_{b}^{2}\right)}=2 \mathrm{~m} \cdot \mathrm{s}^{-1}$ ). It can be seen that $\mathrm{QC}$ using the Gaussian distribution can cause a large number of observations to be unusable in typhoon center areas, while these observations often contain key information of the typhoon structure.

The actual situation is that the deviation between observation and background does not strictly follow the Gaussian distribution, as shown in Figure 5, which demonstrates the deviation distribution of the $u, v$ component of the joint wind field and its best Gaussian fitting curve. In the figure, the true distribution of the $u$ component deviation shows a very obvious asymmetry, and the Gaussian curve does not fit the distribution well. In addition, it can be seen from the figure that there is a certain bias between the joint wind field and the background, so it is necessary to undertake the bias correction process before assimilation (the cause of the bias correction was not analyzed in this study). 


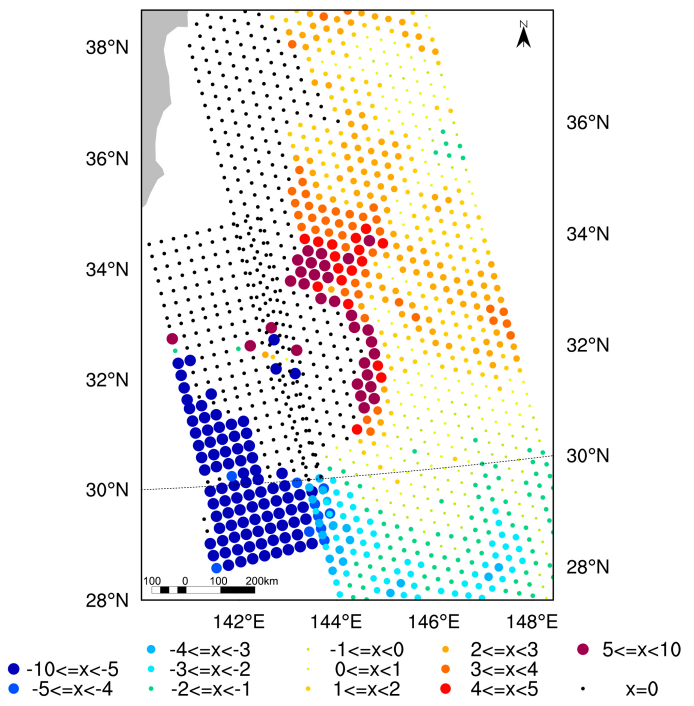

(a)

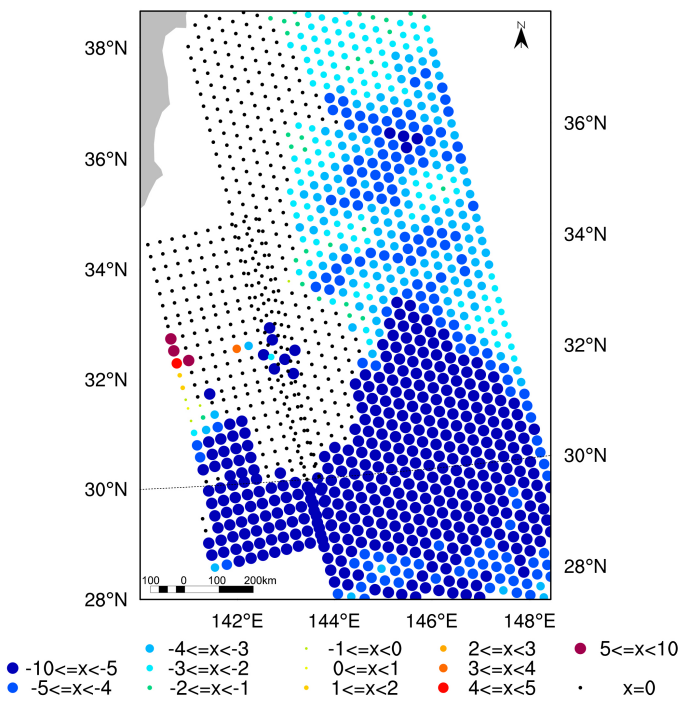

(b)

Figure 4. Gaussian distribution QC of the joint wind field during assimilation, where $x$ represents the magnitude of the deviation $(x=o-b)$, and the black dots indicate the rejected observations: (a) $u$ component; (b) $v$ component.

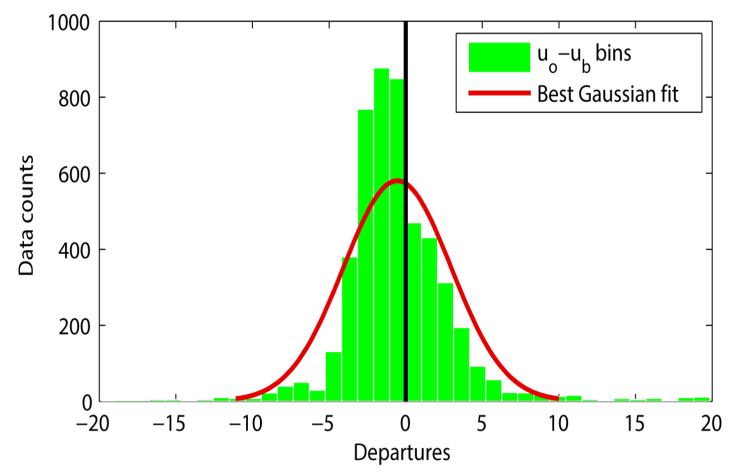

(a)

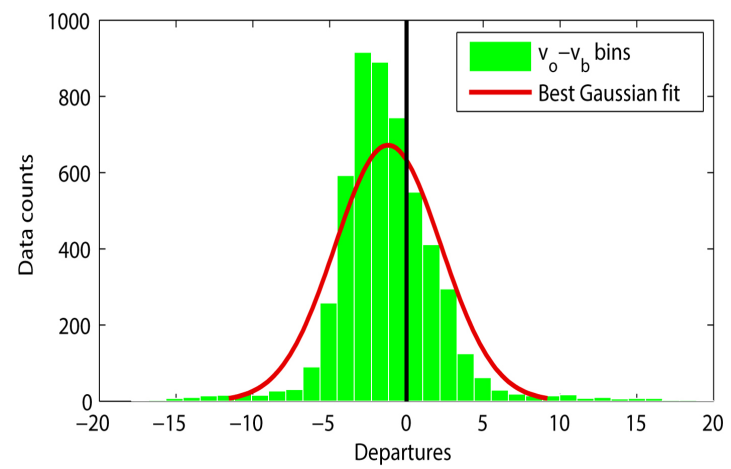

(b)

Figure 5. Best Gaussian fit to the observation deviation distribution: (a) $u$ component; (b) $v$ component.

\subsection{Huber Norm Distribution QC Scheme}

\subsubsection{Definition of Huber Norm}

Research shows that the Huber norm distribution is more consistent with the deviation distribution of the actual observations (Tavolato and Isaksen, 2014). The Huber norm uses a combination of the Gaussian distribution with an exponential distribution, where the Gaussian fit is used for the middle part of the distribution, while the exponential fit is used on both sides, as shown in the equation:

$$
f(x)=\frac{1}{\sigma_{o} \sqrt{2 \pi}} \cdot e^{-\frac{\rho(x)}{2}}
$$

where:

$$
\rho(x)= \begin{cases}\frac{x^{2}}{\sigma_{o}^{2}} & \text { if }|x| \leq c \\ \frac{2 c|x|-c^{2}}{\sigma_{o}^{2}} & \text { if }|x|>c\end{cases}
$$


In the assimilation system, $x=y-H\left(x_{b}\right)$, where $y$ represents the observation, $x_{b}$ is the background field, $H$ is the observation operator and $\sigma_{o}$ represents the observation error. $c$ is the transition point where the Gaussian fitting curve connects with the exponentially fitting curve, that is where the Gaussian fitting curve ends and the exponential fitting curve begins. This definition ensures that the derivative of the function $f$ and $f$ itself are continuous. Since the distribution of the deviation is not strictly symmetrical, the left transition point $c_{L}$ of the function can be different from the right transition point $c_{R}$, and different parameter values are selected according to the type of observation.

\subsubsection{Calculation of the Optimal Transition Point}

Before calculating the transition point of the Huber norm fitting curve for the joint wind field, it is necessary to correct the bias of the observation (this is not discussed in detail here). The Huber norm fitting curve is mainly determined by the parameters $\sigma_{o}, c_{L}, c_{R}$, where $\sigma_{o}$ is known, and $c_{L}, c_{R}$ can be calculated separately. For the calculation of the transition point, it is usually done by searching the value between interval [0.0,5.0] with a given step size of 0.1 [38], where the optimal value of the transition point is the one that has the minimum misfit error between the data distribution and the given Huber norm fitting curve. For each $\left(c_{L}, c_{R}\right)$ pair, the misfit between the Huber norm curve and the data distribution is defined as:

$$
M\left(c_{L}, c_{R}\right)=\sum_{i=1}^{n}\left(\frac{N_{i}}{N_{\text {sum }}}-\frac{S_{i}}{S_{f}}\right)^{2}
$$

where $N_{i}$ is the population in range bin $i$ and $N_{\text {sum }}$ is the number of all data, $S_{i}$ is the integration in range bin $i$ and $S_{f}$ is the integration of function $f(x)$ with the specific Huber distribution (since $f(x)$ is a density function, so its integration $S_{f}$ equals one).

For the $u, v$ component of the joint wind field, we calculate the transition points of the Huber norm distribution for them separately. The optimal Huber norm fitting curve is shown in Figure 6, where the optimal left and right transition point values of the $u$ component are $(1.4,1.2)$, and the optimal left and right transition points of the $v$ component are $(0.6,0.8)$. It can be seen from the figure that the Huber norm curve can better fit the distribution of observation deviation compared to the Gaussian fitting curve.

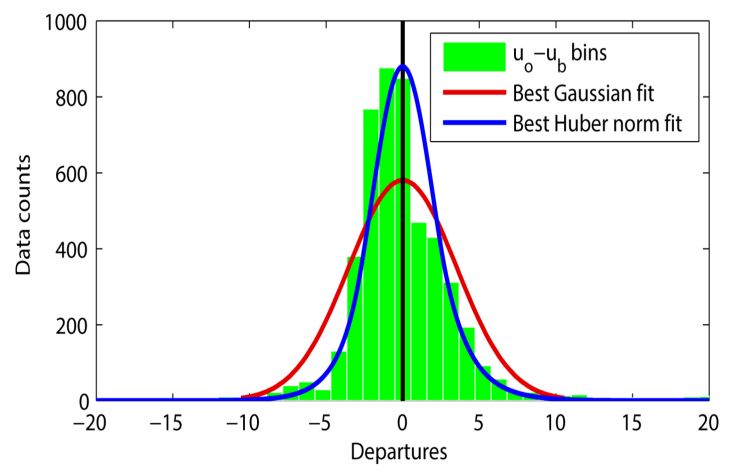

(a)

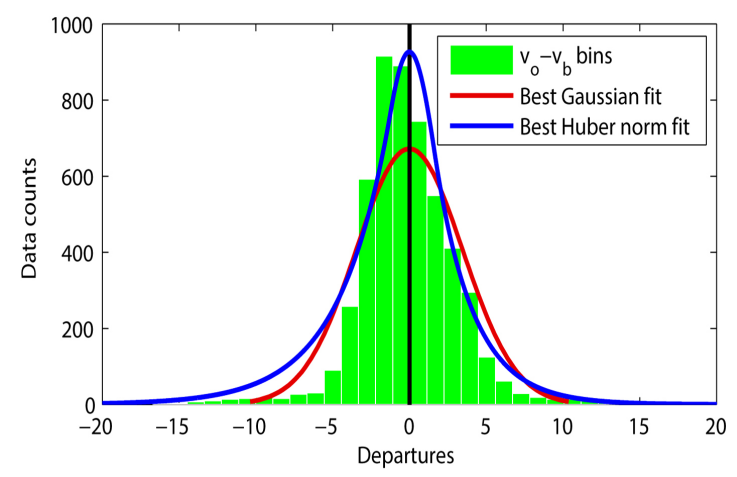

(b)

Figure 6. Best Huber norm fit to the observation deviation distribution: (a) $u$ component; (b) $v$ component.

\subsubsection{Definition of Observation Weight}

The traditional Gaussian distribution QC scheme gives full weight to the observation satisfying the condition $(o-b)^{2}<\alpha^{2}\left(\sigma_{o}^{2}+\sigma_{b}^{2}\right)$. For the Huber norm distribution QC scheme, the main purpose is to give a more reasonable weight to the observation. Observation with a smaller deviation to the background is given a larger weight, while observation with a bigger deviation is given a smaller 
weight. By this mean, it makes sure that observation with a big deviation can still affect the final analysis, while ensuring the stability of the assimilation process.

The cost function of the QC for a single observation is [39]:

$$
J_{O}^{Q C}=-\frac{1}{2} \ln (f(x))=\rho(x)+\text { const }
$$

For the weight attached to a single observation, the value of the weight is given by the ratio of $J_{0}^{\mathrm{QC}}$ to its cost function under the Gaussian assumption, namely:

$$
W=\frac{J_{0}^{Q C}}{J_{0}^{\text {Gaussian }}}
$$

When $|x|<c\left(\right.$ or $\left.-c_{L} \leq x \leq c_{R}\right)$,

$$
W=\frac{2 \ln \left(\sigma_{o} \sqrt{2 \pi}\right)+\left(\frac{y-h\left(x_{b}\right)}{\sigma_{o}}\right)^{2}}{2 \ln \left(\sigma_{o} \sqrt{2 \pi}\right)+\left(\frac{y-h\left(x_{b}\right)}{\sigma_{o}}\right)^{2}}=1
$$

When $|x|>c\left(\right.$ or $x<-c_{L}$ or $\left.x>c_{R}\right)$,

$$
W=\frac{2 \ln \left(\sigma_{o} \sqrt{2 \pi}\right)-\frac{c^{2}}{\sigma_{o}^{2}}+\frac{2 c\left|y-h\left(x_{b}\right)\right|}{\sigma_{o}^{2}}}{2 \ln \left(\sigma_{o} \sqrt{2 \pi}\right)+\left(\frac{y-h\left(x_{b}\right)}{\sigma_{o}}\right)^{2}}
$$

Since $|x|>c$,

$$
(c-|x|)^{2}=c^{2}-2 c\left|y-h\left(x_{b}\right)\right|+\left(y-h\left(x_{b}\right)\right)^{2}>0
$$

which is:

$$
\left(y-h\left(x_{b}\right)\right)^{2}>-c^{2}+2 c\left|y-h\left(x_{b}\right)\right|
$$

we can conclude that $W<1$, that is to say, it reduces the weight of observation when the deviation drops out of the transition point.

Figure 7 shows the weight assigned to observation using the Gaussian distribution QC scheme and the Huber norm distribution QC scheme. As shown in the figure, the observation whose deviation drops between the two transition points is given equal weight, while the weight of others is reduced by the ratio of $J_{o}^{Q C}$ to its cost function under the Gaussian assumption.

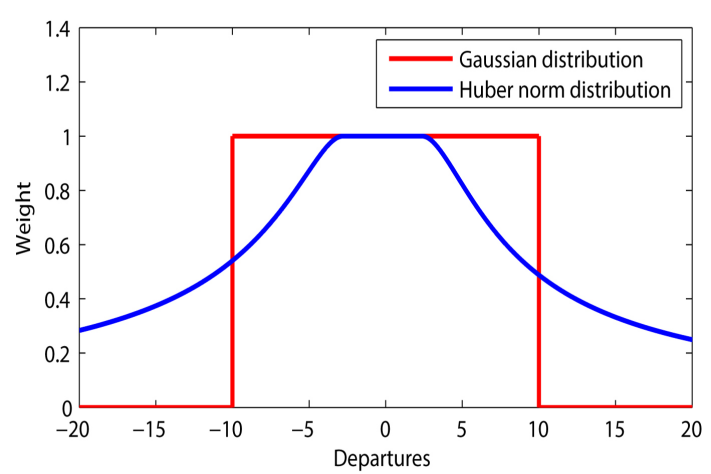

(a)

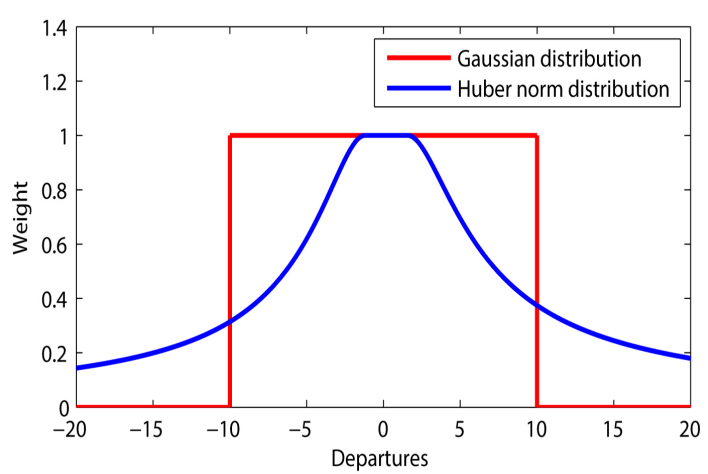

(b)

Figure 7. The corresponding weights after applying the variational QC. Red line: Gaussian distribution; blue line: Huber norm distribution: (a) $u$ component; (b) $v$ component. 


\section{Assimilation Experiments}

\subsection{Experimental Setup}

In this study, we select typhoon Lionrock (2016) as a numerical example, which was generated in the Northwest Pacific at 2000 UTC 19 August 2016, in the vicinity of $\left(33.1^{\circ} \mathrm{N}, 141.4^{\circ} \mathrm{E}\right)$. The center's maximum wind speed was about $18 \mathrm{~m} \cdot \mathrm{s}^{-1}$, and its pressure was about $994 \mathrm{hPa}$. Lionrock weakened to an extratropical cyclone on 31 August. The the Weather Research and Forecasting model data assimilation system (WRFDA) developed by the National Center for Atmospheric Research (NCAR) [40] is adopted in this study. The WRFDA system is a widely-used operational system that can produce a multivariate incremental analysis in the WRF model space [41]. The grid size of the assimilation region is $260 \times 250$; the horizontal resolution is $15 \mathrm{~km}$; and the vertical discretization is 30 layers. The time of assimilation is based on the time window of the joint wind field of the typhoon region, which was 0900 UTC 29 August 2016. The NCEP FNL (Final) Operational Global Analysis data are used as the initial field and boundary conditions. This product is on one-degree by one-degree grids prepared operationally every six hours. It is from the Global Data Assimilation System (GDAS), which continuously collects observational data from the Global Telecommunications System (GTS) and other sources, for many analyses. We take the 21-h forecast adjustment from 1200 UTC 28 August 2016 to 0900 UTC 29 August 2016 as the background field of the assimilation system. After the assimilation, a 30-h forecast is made, which is a forecast to 1500 UTC 30 August 2016.

In this study, a set of assimilation and comparison experiments is carried out. The assimilation experimental design is shown in Table 1, in terms of the QC scheme (traditional QC and Huber norm QC) and observation types (SAR wind, ASCAT wind and joint wind). The control experiment is just a forecast of the background field without assimilation. The purpose of Experiments 1, 2 and 4 is to show if the assimilation of joint wind improves the analysis compared to the single wind type scheme, while the aim of Experiments 3 and 4 is to show if the Huber norm method gives a better result than the traditional QC scheme.

Table 1. Data assimilation experimental design.

\begin{tabular}{ccc}
\hline Experiment Name & QC Scheme & Observation Type \\
\hline 1 & Huber norm distribution QC & SAR wind \\
2 & Huber norm distribution QC & ASCAT wind \\
3 & Gaussian distribution QC & SAR + ASCAT wind \\
4 & Huber norm distribution QC & SAR + ASCAT wind \\
\hline
\end{tabular}

\subsection{Experimental Results and Discussion}

We take the FNL data at the analysis time as the reference (close to the truth, but not the real truth) and to see the analysis errors (analysis minus reference) of different experiments. Figure 8 gives the analysis errors of the pressure field of different experiments at the 10-m height of the center area of typhoon Lionrock at the analysis time. We still see some big analysis errors of different experiments in the typhoon center; however, the joint wind assimilation using the Huber norm distribution QC has the minimum analysis errors of pressure field compared to the others. An accurate initial field (the analysis is used as the initial field for the forecast) is crucial to a good numerical forecast, as can be seen in the following forecast results.

Figure 9a shows the observed typhoon path and forecasted typhoon paths of different experiments. It is apparent that the location of the typhoon center based on different QC schemes is very close to the control experiment (with no assimilation of the scatterometer wind) at the time of the assimilation, and this may due the defect of the position algorithm of the typhoon center. However, the forecasted typhoon paths of wind assimilation all show some improvement compared to that of the control experiment. The time indicators in Figure 9a demonstrate that the joint wind assimilation using the Huber norm distribution QC has the best forecasted path. In order to better compare the accuracy 
of the assimilation experiments with the typhoon moving path, Figure $9 \mathrm{~b}$ gives the error of the forecasted typhoon path. As Figure 9 shows, with an increase in forecast time, the typhoon path error of most of the assimilation experiments becomes obviously smaller than that of the control experiment. The experiment with SAR wind, however, shows some large errors after a forecast of $27 \mathrm{~h}$, mainly because that SAR wind alone contains no new direction information of wind, and the amount of data is quite small after thinning to a resolution of $25 \mathrm{~km}$. We get the benefit from the joint assimilation of SAR wind and ASCAT wind comparing to the assimilation of a single type of wind, and it improves the typhoon forecasted path using the Huber norm distribution QC compared to the Gaussian distribution QC scheme.

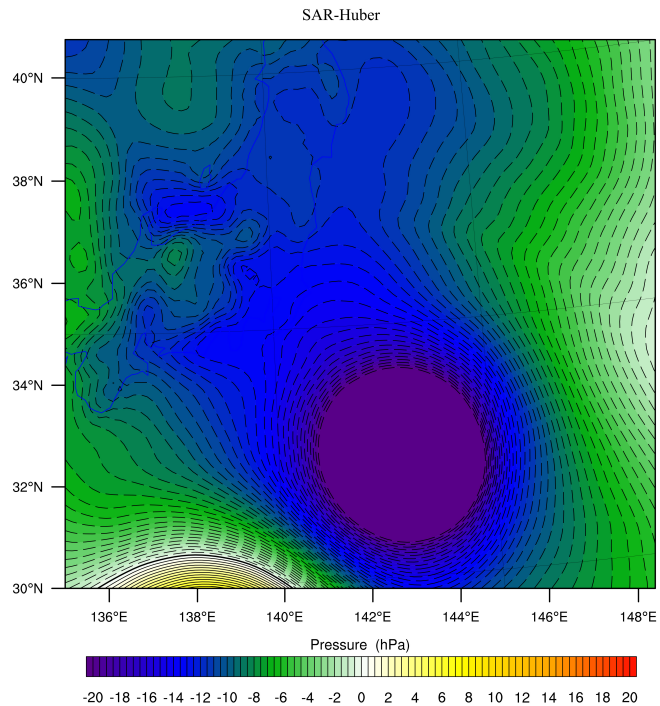

(a)

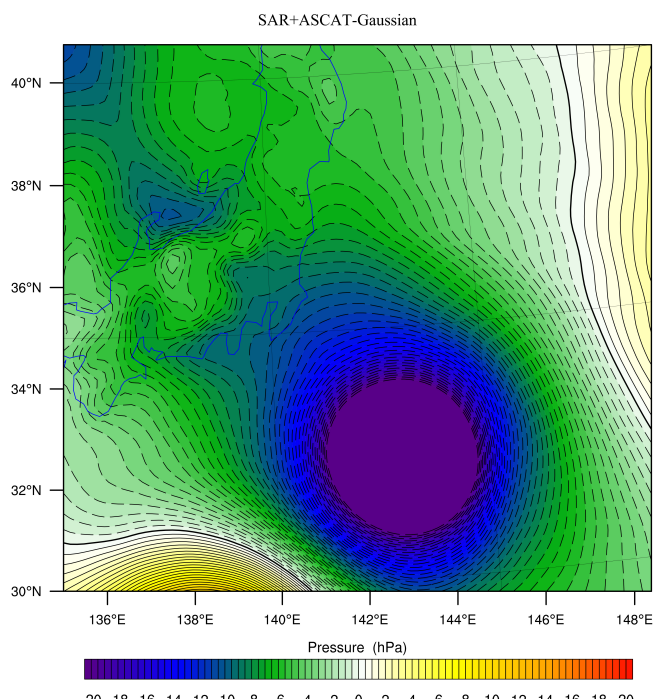

(c)

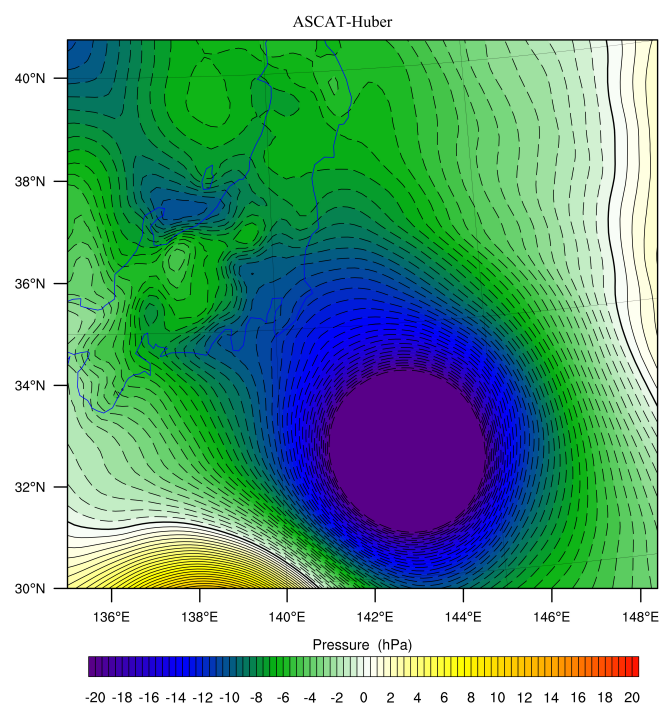

(b)

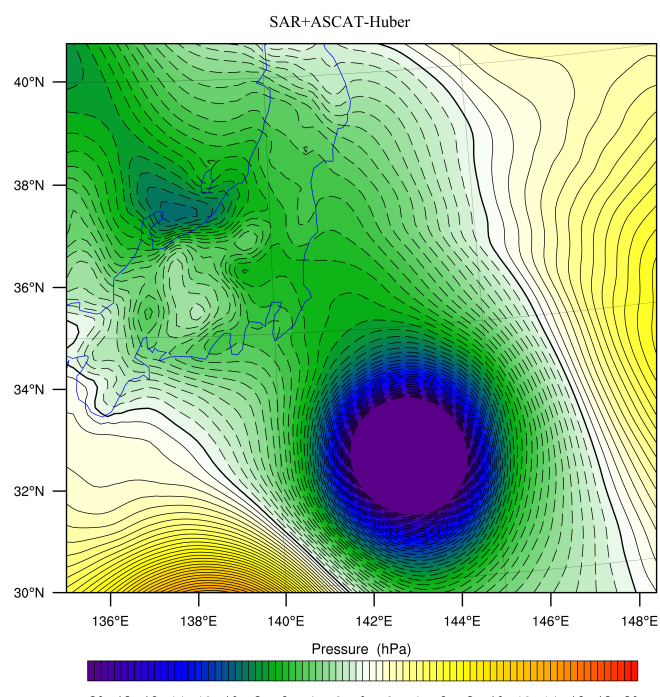

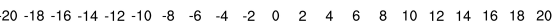

(d)

Figure 8. Pressure error of the center area of typhoon Lionrock at the analysis time: (a) assimilation of SAR wind using the Huber norm distribution QC; (b) assimilation of ASCAT wind using the Huber norm distribution $\mathrm{QC} ;(\mathbf{c})$ assimilation of joint wind using the Gaussian distribution $\mathrm{QC}$; (d) assimilation of joint wind using the Huber norm distribution QC. 


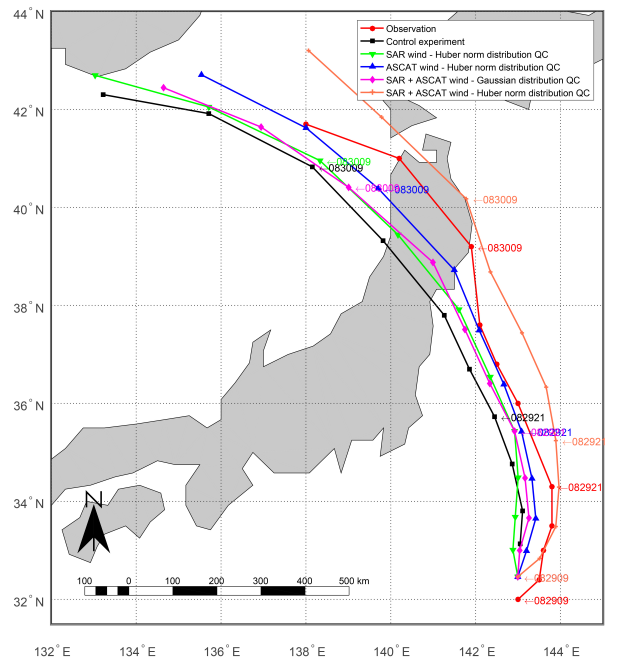

(a)

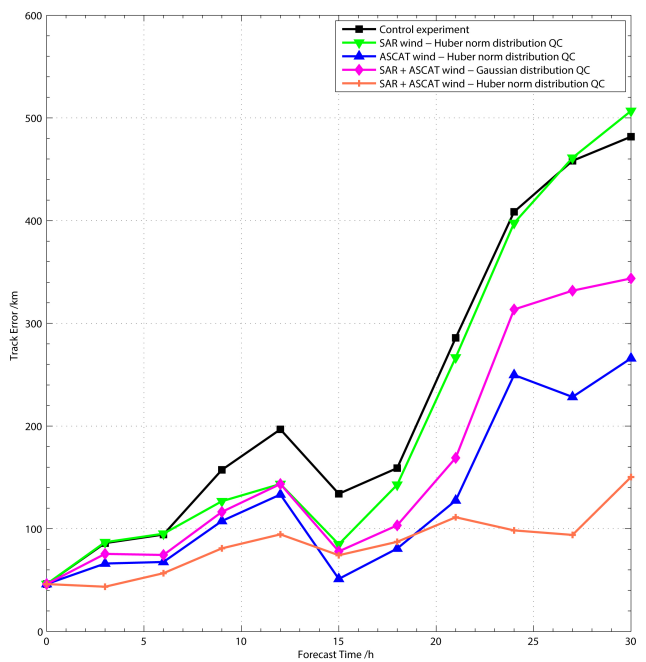

(b)

Figure 9. Forecasted path and track errors of typhoon Lionrock: (a) forecasted path of different experiments and the observed path. The time indicator is given every $12 \mathrm{~h}$. (b) Track errors of different experiments compared to the observed path.

The intensity forecasts of the typhoon based on the different schemes are compared in Figure 10, and Table 2 gives the quantitative analysis of the data assimilation experiments. As shown in the figure, the assimilation of wind data improves the intensity forecast of the typhoon. The joint wind assimilation using the Huber norm, however, in some way weakens the minimum pressure and strengthens the maximum wind speed of the typhoon eye at the time of the assimilation. Although there is a bigger misfit between the intensity forecast and the observed truth than other experiments at the analysis time, it has a better analysis field than the others (see Figure 8). As can be seen from Table 2, the joint assimilation of SAR and ASCAT wind using the Huber norm distribution QC has the minimum average errors on track forecast $(85.24 \mathrm{~km})$ and intensity forecast $\left(-5.61 \mathrm{~m} \cdot \mathrm{s}^{-1}\right.$ for speed and $-1.84 \mathrm{hPa}$ for pressure, respectively). Therefore, the joint wind assimilation using the Huber norm distribution QC has also improved the intensity forecast of the typhoon.

Figures 11 and 12 show the $(O-A)$ (observation minus analysis, namely residual) diagram and $O / A$ (observation / analysis ) comparison of the bias, root-mean-square value and standard derivation of the $u$ and $v$ components of the four experiments. Assimilation experiment with the SAR wind field using Huber norm distribution QC shows a big deviation from the analysis (Figure 11a,e), thus having a big $r m s\left(r m s_{u}=5.46, r m s_{v}=4.50\right)$ for the analysis (Figure 12a,e). This is mainly because most of the wind observations of SAR were located in the center area of the typhoon (as can be seen in Figure 2 ), and about one third of the observations have a deviation more than five-times the observation error to the background $\left(n_{x>5 \sigma}=93, n_{x \leq 5 \sigma}=188\right)$. Observations with a bigger deviation from the background have a smaller weight using the Huber norm distribution QC scheme. Smaller weight in the observation means the analysis gets closer to the background or a bigger residual, as shown in the Figure 11a,e. This also can be seen from Figure 11b,d,f,h, where the residual is much bigger in the center area of the typhoon. While the joint wind assimilation using the Gaussian distribution QC has the minimum $r m s\left(r m s_{u}=1.19, r m s_{v}=1.06\right)$ compared to the others (Figure 12c,g), since observations with a big deviation from the background have been decreased in the QC step (Figure 11c,g), and the remaining observations have an equal weight. 
Table 2. Quantitative analysis of data assimilation experiments.

\begin{tabular}{|c|c|c|c|c|c|c|c|}
\hline Observation Type/QC Scheme & $n_{x \leq 5 \sigma} *$ & $n_{x>5 \sigma}$ & $r m s_{u}^{* *}$ & $r m s_{v}$ & $\overline{e_{\text {track }}} * * *$ & $\overline{e_{\text {speed }}}$ & $\overline{e_{\text {pressure }}}$ \\
\hline SAR/Huber norm & 188 & 93 & 5.46 & 4.50 & 214.44 & -6.43 & 7.05 \\
\hline ASCAT/Huber norm & 4844 & 75 & 1.48 & 1.27 & 129.50 & -6.52 & 7.14 \\
\hline SAR + ASCAT/Gaussian & 4717 & 0 & 1.19 & 1.06 & 163.26 & -6.84 & 6.48 \\
\hline SAR + ASCAT/Huber norm & 4776 & 113 & 3.96 & 1.85 & 85.24 & -5.61 & -1.84 \\
\hline
\end{tabular}

* $n$ is the number of observations used in the assimilation, $x=o-b, \sigma=\sqrt{\sigma_{o}^{2}+\sigma_{b}^{2}}$;

** $r m s$ is the root mean square of $o-a$; the unit is $\mathrm{m} \cdot \mathrm{s}^{-1}$;

$* * * \bar{e}$ is the average forecast error; the units are $\mathrm{km}, \mathrm{m} \cdot \mathrm{s}^{-1}$ and $\mathrm{hPa}$, respectively.

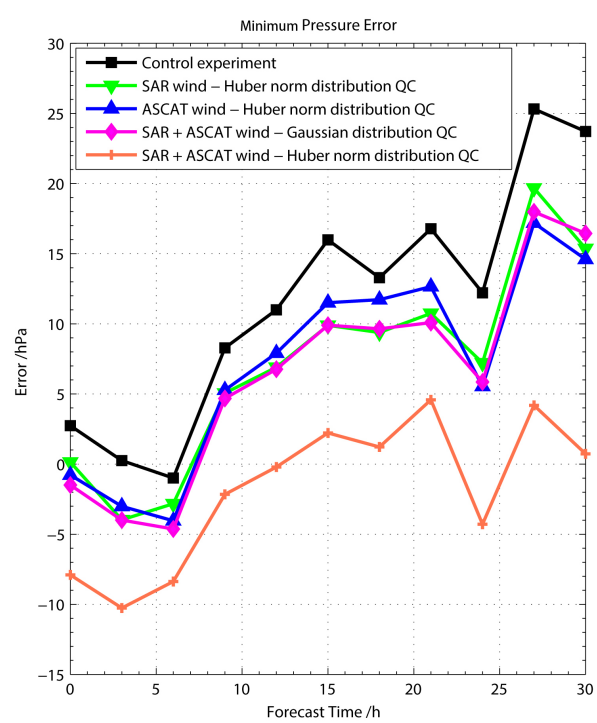

(a)

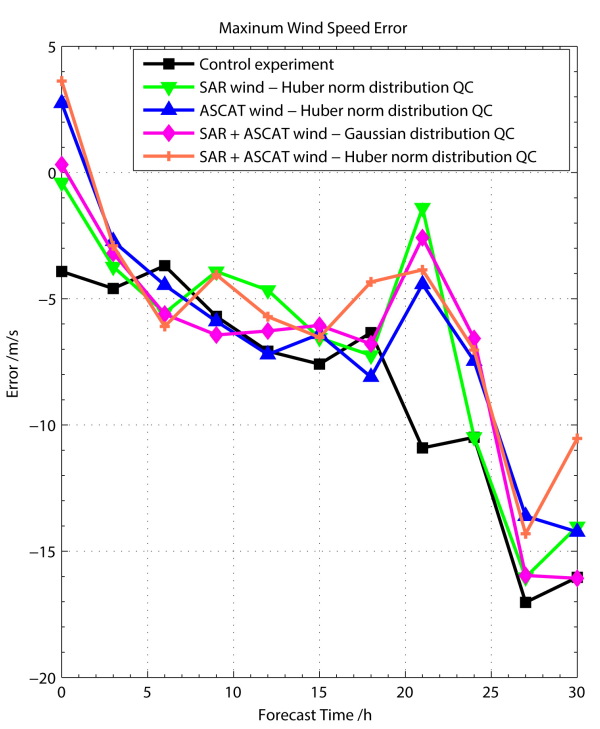

(b)

Figure 10. Intensity forecast errors of the typhoon eye: (a) minimum pressure forecast errors. (b) maximum wind speed forecast errors.

It can be seen from Table 2 that the rms mainly depends on the ratio of the observations with a big deviation to the background $(x>5 \sigma)$ in the wind field. The higher the ratio, the bigger the rms. However, it also can be seen from the table that the effect on the assimilation is not determined by the rms, but the distribution of the observation. The experiment with ASCAT wind and the experiment with joint wind using the Huber norm QC have almost the same ratio as the observations with a big deviation, but the latter has a more complete observation of the typhoon center assimilated in the assimilation step (see Figure $11 \mathrm{~b}, \mathrm{~d}, \mathrm{f}, \mathrm{h}$ ), thus a more positive impact on the typhoon prediction. This is also the truth for the comparison of two different QC schemes (see Figure 11c,d,g,h).

It can be seen from Equation (5) that with the Huber norm distribution applied, the $J_{0}^{Q C}$ is an $L^{2}$ norm in the center of the distribution and an $L^{1}$ norm in the tails (Tavolato and Isaksen, 2014). This makes the Huber norm QC a robust method that allows the use of observations with a large deviation from the background. It also makes it safe to use observations with a few erroneous outliers, since observation with a very large deviation only has a small weight and affects the analysis very little. Figure 13 shows that the QC scheme with the Huber norm has a fast convergence to the minima in the minimization process, as well as the Gaussian distribution QC scheme, which proves that the Huber norm QC is a robust method. 


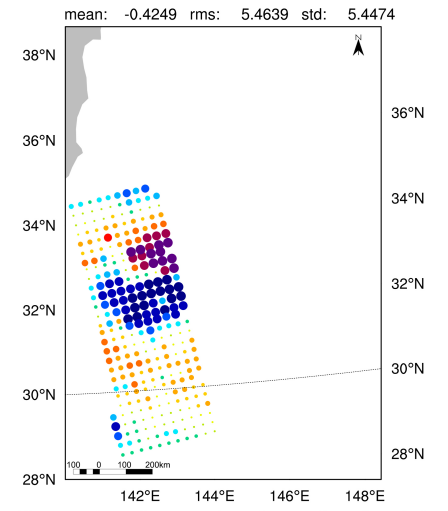

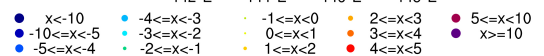

(a)

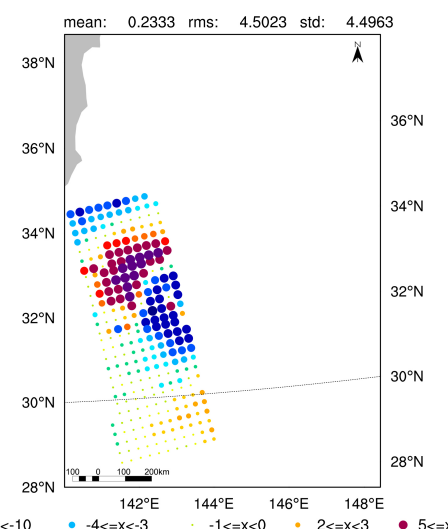

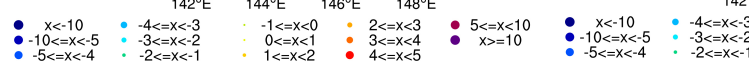

(e)

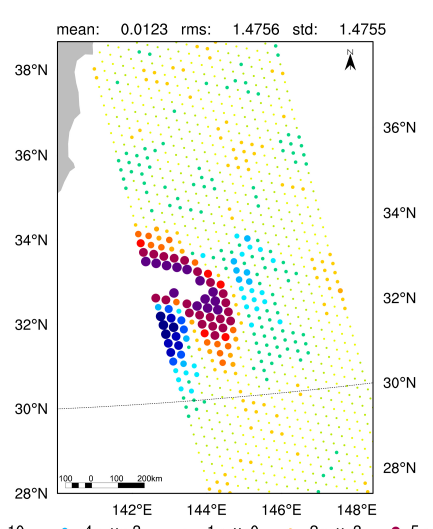

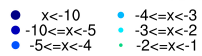

(b)

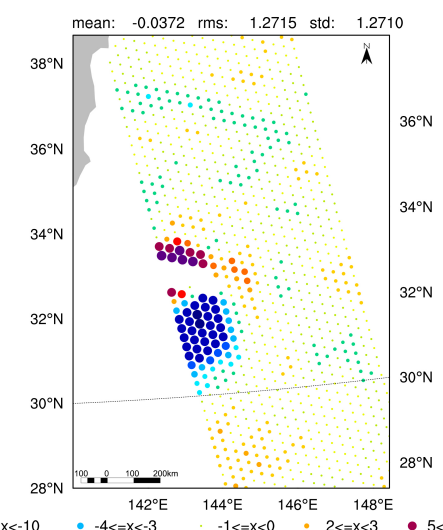

(f)

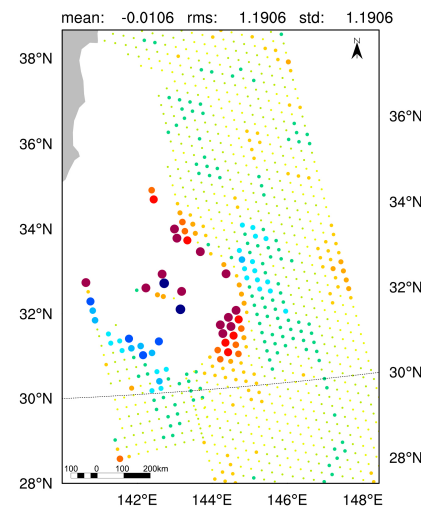

(c)

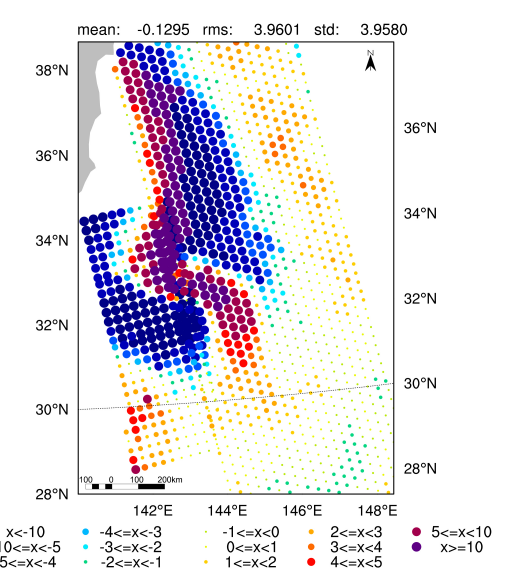

(d)

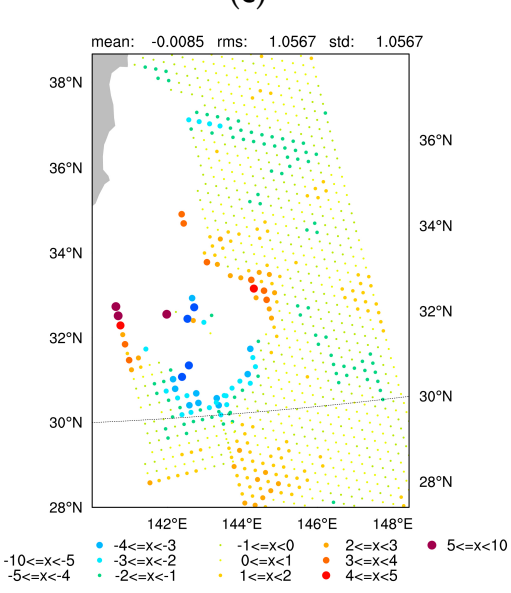

(g)

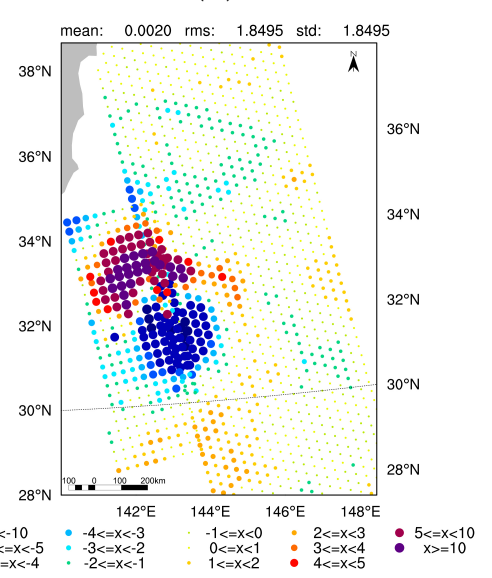

(h)

Figure 11. Observation residual after assimilation, where $x$ represents the magnitude of the residual $(x=o-a)$ : (a) residual of the $u$ component of SAR wind using the Huber norm distribution QC; (b) residual of the $u$ component of ASCAT wind using the Huber norm distribution QC; (c) residual of the $u$ component of joint wind using the Gaussian distribution QC; (d) residual of the $u$ component of joint wind using the Huber norm distribution QC; (e) residual of the $v$ component of SAR wind using the Huber norm distribution QC; (f) residual of the $v$ component of ASCAT wind using the Huber norm distribution QC; (g) residual of the $v$ component of joint wind using the Gaussian distribution QC; (h) residual of the $v$ component of joint wind using the Huber norm distribution QC. 


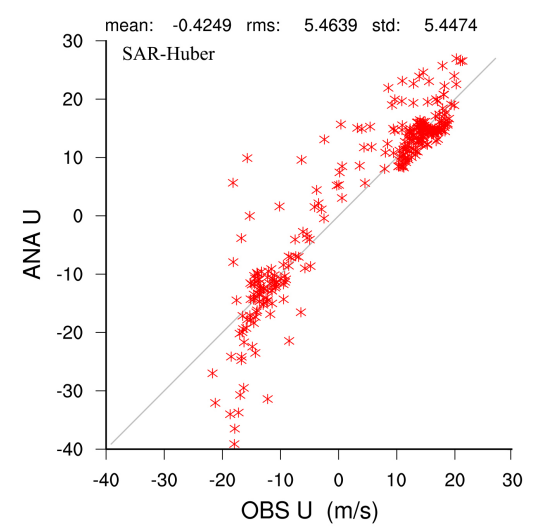

(a)

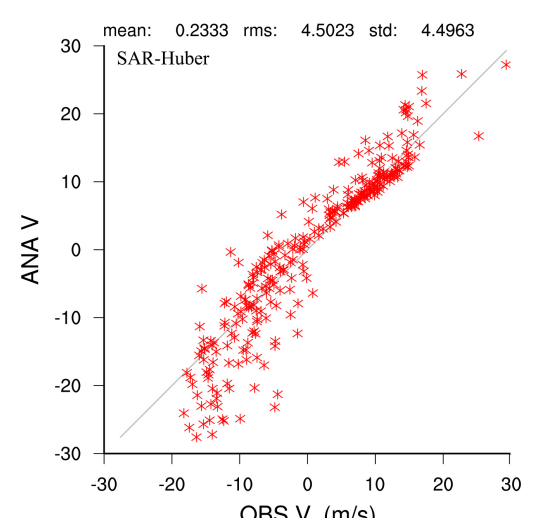

(e)

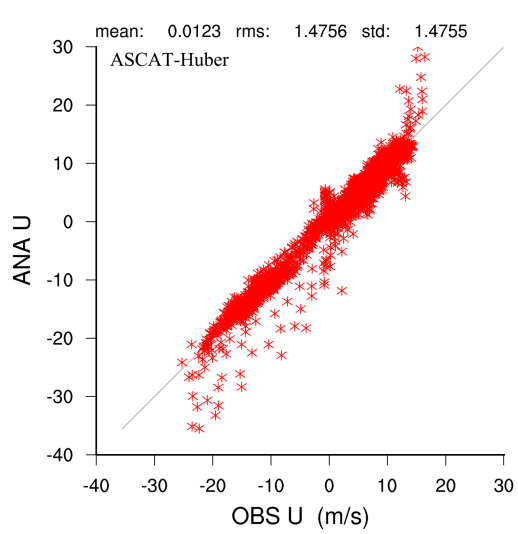

(b)

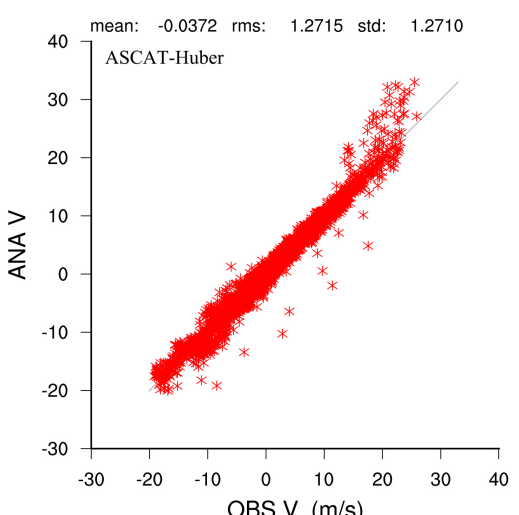

(f)

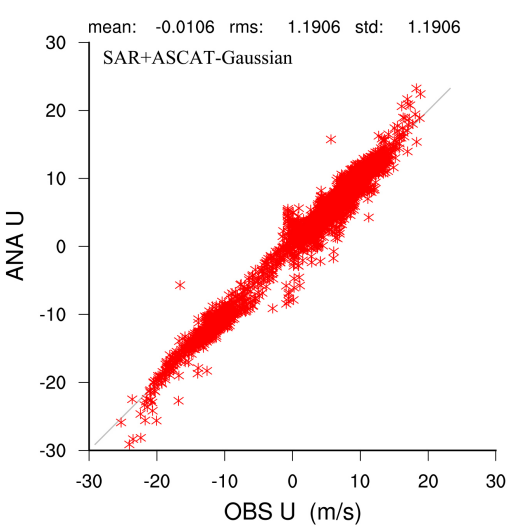

(c)

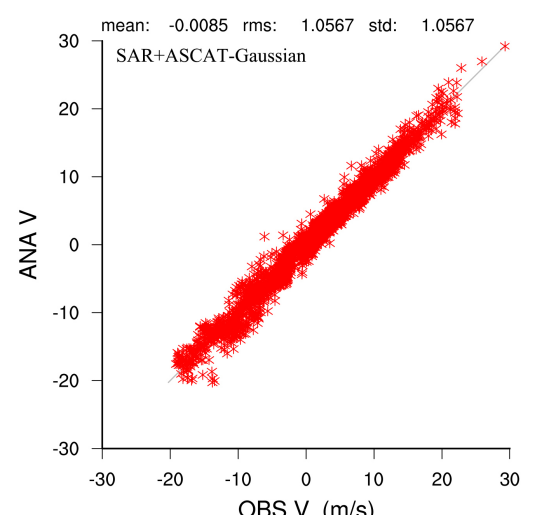

(g)

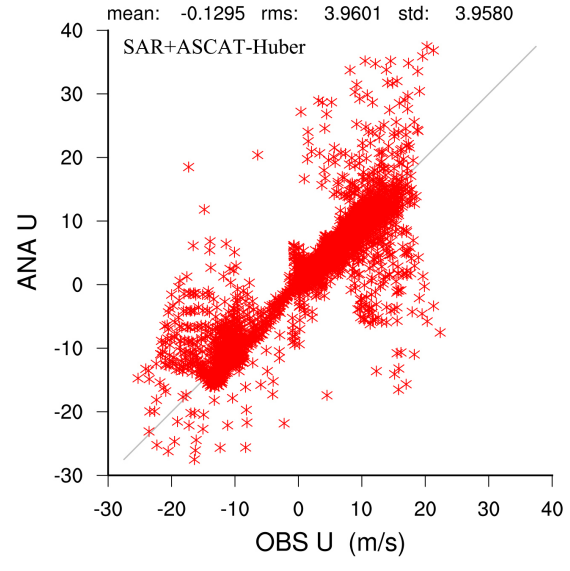

(d)

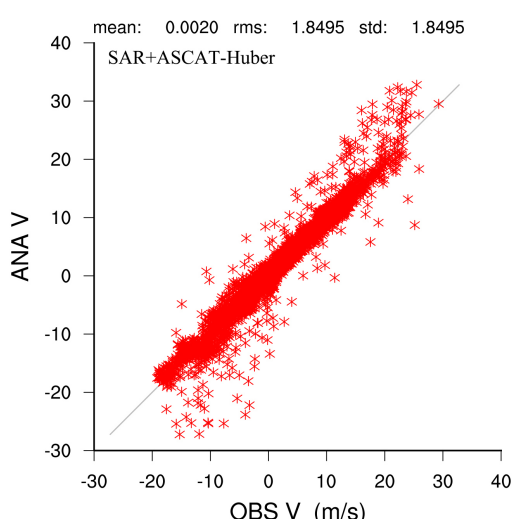

(h)

Figure 12. $O / A$ (observation / analysis) comparison of the bias, root-mean-square value and standard derivation of the $u$ and $v$ wind components: (a) $u$ component of SAR wind using the Huber norm distribution QC; (b) $u$ component of ASCAT wind using the Huber norm distribution QC; (c) $u$ component of joint wind using the Gaussian distribution QC; (d) $u$ component of joint wind using the Huber norm distribution QC; (e) $v$ component of SAR wind using the Huber norm distribution QC; (f) $v$ component of ASCAT wind using the Huber norm distribution QC; (g) $v$ component of joint wind using the Gaussian distribution QC; (h) $v$ component of joint wind using the Huber norm distribution QC. 


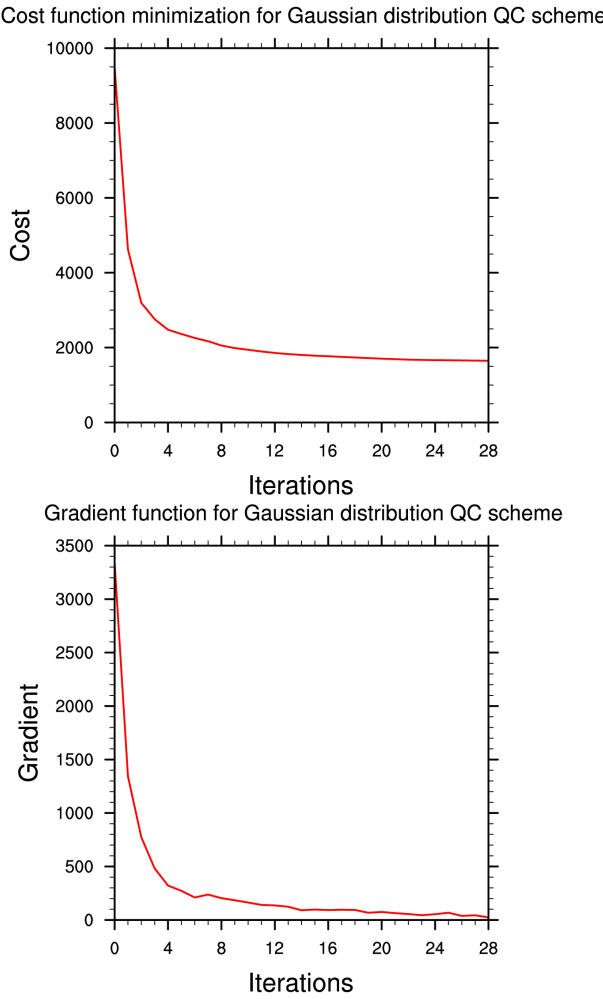

(a)
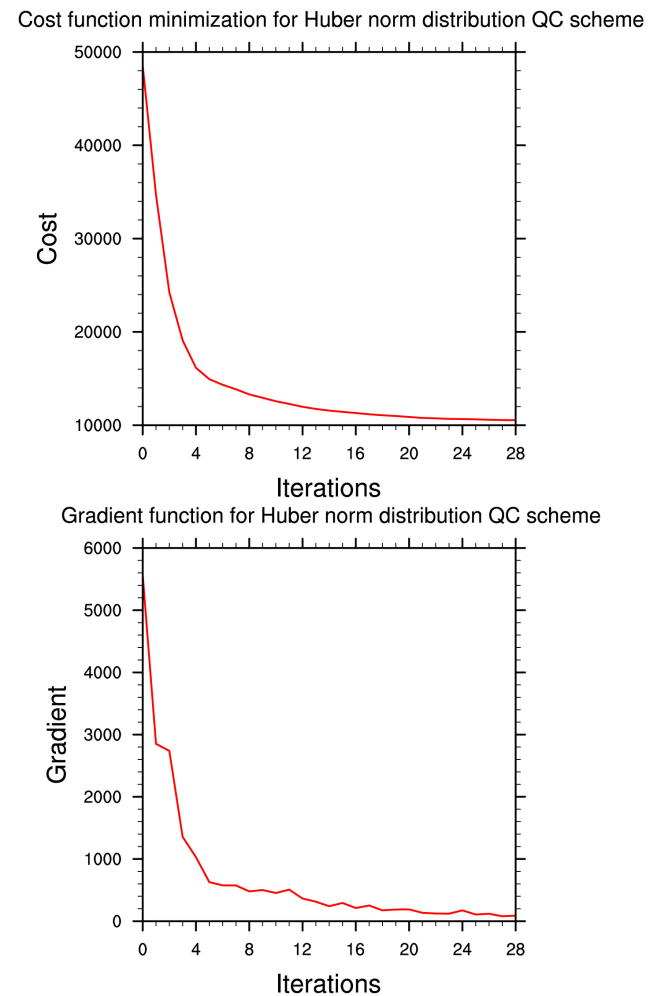

(b)

Figure 13. Minimization process of the cost function and the gradient for assimilation of the joint wind field: (a) Gaussian distribution QC scheme; (b) Huber norm distribution QC scheme.

However, although the joint wind field provides a detailed wind field of the typhoon, the information of the wind in the center area of the typhoon is not fully used with the Huber norm QC, since observation only have a small weight. This is mainly due to the bad background, which usually cannot provide the high speed wind structure of the typhoon.

We can see from the results that when a detailed wind field is available for the typhoon, a more accurate analyze was obtained when the Huber norm QC was applied, thus a more accurate prediction for the typhoon.

\section{Conclusions}

Based on NCAR's WRFDA system, we conducted a joint assimilation experiment of the ASCAT wind and SAR wind for the typhoon "Lionrock (2016)" and introduced the Huber norm QC scheme. Combining the SAR wind field with the ASCAT wind field, we take advantage of both observations, which not only make up the small swath limit of the SAR wind, but also fill the vacancy of the ASCAT wind field in the typhoon area. The results of assimilation experiments show that the joint assimilation improved the typhoon track forecast results. In addition, the Huber norm distribution QC scheme is adopted to increase the usage of the observation in the typhoon center area and to assign more reasonable weight to the observation, thus improving the analysis.

Although VH polarization observation of SAR can retrieve high speed wind, the impact on typhoon forecast is limited due to the quality of the background and parameterization scheme of the typhoon model. On the one hand, the background wind field of the typhoon is generally smoother than the real typhoon field, resulting in big deviation for the high speed wind observation, which led to the small weight of observation in the process of assimilation. On the other hand, since the parameterization of the typhoon model is not optimal, the mechanism of high speed wind for the 
development of the typhoon is not clear. Therefore, the improvement of the parameterization of the typhoon model is the next step we should consider.

Acknowledgments: This work was supported by the Key Research and Development Program of Hainan Province (ZDYF2017167) and the projects of the National Natural Science Foundation (NSFC40775064 and NSFC41675097).

Author Contributions: Boheng Duan, Weimin Zhang, Xiaofeng Yang and Yi Yu initiated the research. Under supervision of Weimin Zhang and Xiaofeng Yang, Boheng Duan performed the experiments and analysis. Boheng Duan, Weimin Zhang, Xiaofeng Yang and Haijin Dai wrote and revised the manuscript. All authors read and approved the final version of the manuscript.

Conflicts of Interest: The authors declare no conflict of interest.

\section{References}

1. Li, B.; Jung, J.A.; Morgan, M.C. Assessment of Assimilating ASCAT Surface Wind Retrievals in the NCEP Global Data Assimilation System. Mon. Weather Rev. 2011, 139, 3405-3421.

2. Isaksen, L.; Janssen, P.A. Impact of ERS scatterometer winds in ECMWF's assimilation system. Q. J. R. Meteorol. Soc. 2004, 130, 1793-1814.

3. Stoffelen, A.; Cats, J.C. The impact of Seasat-A scatterometer data on high-resolution analyses and forecasts: The development of the QEII storm. Mon. Weather Rev. 1991, 119, 2794-2802.

4. Stoffelen, A.; Beukering, P.V. The Impact of Improved Scatterometer Winds on HIRLAM Analyses and Forecasts. HIRLAM Technical Report 31. HIRLAM. Available online: http://hirlam.org/ (accessed on 1 January 2017).

5. Isaksen, L.; Stoffelen, A. ERS scatterometer wind data impact on ECMWF's tropical cyclone forecasts. IEEE Trans. Geosci. Remote Sens. 2000, 38, 1885-1892.

6. Atlas, R.; Hoffman, R.N. The Use of Satellite Surface Wind Data to Improve Weather Analysis and Forecasting at the NASA Data Assimilation Office; Elsevier Oceanography Series; Elsevier: New York, NY, USA, 2000; pp. 57-78.

7. Candy, B. The Assimilation of Ambiguous Scatterometer Winds Using a Variational Technique: Method and Forecast Impact; Met Office, NWP Division: Berkshire, UK, 2001.

8. Atlas, R.; Hoffman, R.N.; Leidner, S.M.; Sienkiewicz, J. The effects of marine winds from scatterometer data on weather analysis and forecasting. Bull. Am. Meteorol. Soc. 2001, 82, 1965-1990.

9. Andersson, E.; Haseler, J.; Undén, P. The ECMWF implementation of three-dimensional variational assimilation (3D-Var). III: Experimental results. Q. J. R. Meteorol. Soc. 1998, 124, 1831-1860.

10. Prasad, S.V.; Gupta, A.; Rajagopal, E.N. Impact of OSCAT surface wind data on T574L64 assimilation and forecasting system-A study involving tropical cyclone Thane. Curr. Sci. 2013, 104, 627-631.

11. Hersbach, H. Assimilation of Scatterometer Data as Equivalent-Neutral Wind; ECMWF Publications: Reading, UK, 2010.

12. Francois, C.; Quesney, A.; Ottle, C. Sequential Assimilation of ERS-1 SAR data into a doupled land surface-hydrological model using an extended kalman filter. J. Hydrometeorol. 2003, 4, 473-487.

13. Matgen, P.; Montanari, M.; Hostache, R.; Pfister, L.; Hoffmann, L.; Plaza, D.; Pauwels, V.R.N.; De Lannoy, G.J.M.; Keyser, R.D.; Savenije, H.H.G. Towards the sequential assimilation of SAR-derived water stages into hydraulic models using the particle filter: Proof of Concept. Hydrol. Earth Syst. Sci. 2010, 14, 1773-1785.

14. Scott, A.K.; Ashouri, Z.; Buehner, M.; Pogson, L.; Carrieres, T. Assimilation of ice and water observations from SAR imagery to improve estimates of sea ice concentration. Tellus A 2015, 67, doi:10.3402/tellusa.v67.27218.

15. Phan, V.X.; Ferro-Famil, L.; Gay, M.; Durand, Y.; Dumont, M.; Morin, S.; Allain, S.; D’Urso, G.; Girard, A. 1D-Var multilayer assimilation of X-band SAR data into a detailed snowpack model. Cryosphere 2014, 8, 1975-1987.

16. Pichelli, E.; Ferretti, R.; Cimini, D.; Panegrossi, G.; Perissin, D.; Pierdicca, N.; Rocca, F.; Rommen, B. InSAR water vapor data assimilation into mesoscale model MM5: Technique and pilot study. IEEE J. Sel. Top. Appl. Earth Obs. Remote Sens. 2015, 8, 3859-3875.

17. Mason, C.D.; Garcia-Pintado, J.; Cloke, H.L.; Dance, S.L. Evidence of a topographic signal in surface soil moisture derived from ENVISAT ASAR wide swath data. Int. J. Appl. Earth Obs. Geoinf. 2016, 45, 178-186. 
18. Li, X.F.; Pichel, W.G.; He, M.X.; Wu, S.Y. Observation of hurricane-generated ocean swell refraction at the Gulf Stream north wall with the RADARSAT-1 synthetic aperture. IEEE Trans. Geosci. Remote Sens. 2002, 40, 2131-2142.

19. Li, X.F.; Zhang, J.A.; Yang, X.F.; Pichel, W.G. Tropical Cyclone Morphology from Spaceborne Synthetic Aperture Radar. Bull. Am. Meteorol. Soc. 2013, 94, 215-230.

20. Jin, S.H.; Wang, S.; Li, X.F. Typhoon eye extraction with an automatic SAR image segmentation method. Int. J. Remote Sens. 2014, 35, 11-12.

21. Li, X.F. The first Sentinel-1 SAR image of a typhoon. Acta Oceanol. Sin. 2015, 34, 1-2.

22. Monaldo, F.M.; Jackson, C.R.; Pichel, W.G.; Li, X.F. A Weather Eye on Coastal Winds. Eos 2015, 96, 18-19.

23. Lee, I.K.; Shamsoddini, A.; Li, X.F.; Trinder, J.C. Extracting hurricane eye morphology from spaceborne SAR images using morphological analysis. ISPRS J. Photogramm. Remote Sens. 2016, 117, 115-125.

24. Zheng, G.; Yang, J.S.; Liu, A.K.; Li, X.F. Comparison of Typhoon Centers from SAR and IR Images and Those from Best Track Data Sets. IEEE Trans. Geosci. Remote Sens. 2016, 54, 1000-1012.

25. Zhou, X.; Yang, X.F.; Li, Z.W.; Yang, Y. Estimation of tropical cyclone parameters and wind fields from SAR images. Sci. China Earth Sci. 2013, 56, 1977-1987.

26. Monaldo, F.M.; Li, X.F.; Pichel, W.G.; Jackson, C.R. Ocean Wind Speed Climatology from Spaceborne SAR Imagery. Bull. Am. Meteorol. Soc. 2014, 95, 565-569.

27. Zhang, B.; Li, X.F.; Perrie, W.; He, Y.J. Synergistic measurements of ocean winds and waves from SAR. J. Geophys. Res. Oceans 2015, 120, 6164-6184.

28. Hwang, P.A.; Li, X.F.; Zhang, B. Retrieving Hurricane Wind Speed from Dominant Wave Parameters. IEEE J. Sel. Top. Appl. Earth Obs. Remote Sens. 2017, 10, 2589-2598.

29. Yang, X.F.; Li, X.F.; Pichel, W.G.; Li, Z.W. Comparison of Ocean Surface Winds from ENVISAT ASAR, MetOp ASCAT Scatterometer, Buoy Measurements, and NOGAPS Model. IEEE Trans. Geosci. Remote Sens. 2011, $49,4743-4750$.

30. Yang, X.F.; Li, X.F.; Zheng, Q.N.; Gu, X.F. Comparison of Ocean-Surface Winds Retrieved from QuikSCAT Scatterometer and Radarsat-1 SAR in Offshore Waters of the U.S. West Coast. IEEE Geosci. Remote Sens. Lett. 2011, 8, 163-167.

31. Monaldo, F.M.; Jackson, C.; Li, X.F.; Pichel, W.G. Preliminary Evaluation of Sentinel-1A Wind Speed Retrievals. IEEE J. Sel. Top. Appl. Earth Obs. Remote Sens. 2016, 9, 2638-2642.

32. Zhang, G.S.; Perrie, W.; Li, X.F.; Zhang, J.A. A Hurricane Morphology and Sea Surface Wind Vector Estimation Model Based on C-Band Cross-Polarization SAR Imagery. IEEE Trans. Geosci. Remote Sens. 2017, $55,1743-1751$.

33. Lorenc, C.A.; Hammon, O. Objective quality control of observationsusing Bayesian methods-Theory, and practical implementation. Q. J. R. Meteorol. Soc. 1988, 114, 515-543.

34. Lorenc, C.A. Analysis methods for the quality control of observations. In Proceedings of the ECMWF Workshop on the Use and Quality Control of Meteorological Observations for Numerical Weather Prediction, Reading, UK, 6-9 November 1984; pp. 397-428.

35. Huber, J.P. Robust estimates of a location parameter. Ann. Math. Statist. 1964, 35, 73-101.

36. Huber, J.P. The 1972 Wald Lecture Robust statistics: A review. Ann. Math. Stat. 1972, 43, 1041-1067.

37. Zhang, B.; Perrie, W. Cross-Polarized Synthetic Aperture Radar: A New Potential Measurement Technique for Hurricanes. Bull. Am. Meteorol. Soc. 2012, 93, 531-541.

38. Tavolato, C.; Isaksen, L. On the Use of a Huber Norm for Observation Quality Control in the ECMWF 4D-Var. Q. J. R. Meteorol. Soc. 2015, 141, 1514-1527.

39. Lorenc, A.C. Analysis methods for numerical weather prediction. Q. J. R. Meteorol. Soc. 1986, 112, 1177-1194.

40. Barker, M.D.; Huang, W.; Guo, Y.R. A three-dimensional variational data assimilation system for MM5: Implementation and initial results. Mon. Weather Rev. 2004, 132, 897-914.

41. Zhang, Q.F.; Weng, Y.H.; Sippel, J.A. Cloud-resolving hurricane initialization and prediction through assimilation of Doppler radar observations with an ensemble Kalman filter. Mon. Weather Rev. 2009, $137,2105-2125$.

(C) 2017 by the authors. Licensee MDPI, Basel, Switzerland. This article is an open access article distributed under the terms and conditions of the Creative Commons Attribution (CC BY) license (http:// creativecommons.org/licenses/by/4.0/). 